\title{
Observable signatures of dark photons from supernovae
}

\author{
William DeRocco, ${ }^{a}$ Peter W. Graham, ${ }^{a}$ Daniel Kasen, ${ }^{b, c}$ Gustavo Marques-Tavares ${ }^{a, d}$ \\ and Surjeet Rajendran ${ }^{e}$ \\ ${ }^{a}$ Stanford Institute for Theoretical Physics, Stanford University, \\ Stanford, CA 94305, U.S.A. \\ ${ }^{b}$ Berkeley Center for Theoretical Physics, Department of Physics, University of California, \\ Berkeley, CA 94720, U.S.A. \\ ${ }^{c}$ Lawrence Berkeley National Laboratory, \\ Berkeley, CA 94720, U.S.A. \\ ${ }^{d}$ Maryland Center for Fundamental Physics, Department of Physics, University of Maryland, \\ College Park, MD 20742, U.S.A. \\ ${ }^{e}$ Berkeley Center for Theoretical Physics, Department of Physics, University of California, \\ Berkeley, CA 94720, U.S.A. \\ E-mail: wderocco@stanford.edu, pwgraham@stanford.edu, \\ kasen@berkeley.edu, gusmt@umd.edu, surjeet@berkeley.edu
}

ABSTRACT: A dark photon is a well-motivated new particle which, as a component of an associated dark sector, could explain dark matter. One strong limit on dark photons arises from excessive cooling of supernovae. We point out that even at couplings where too few dark photons are produced in supernovae to violate the cooling bound, they can be observed directly through their decays. Supernovae produce dark photons which decay to positrons, giving a signal in the $511 \mathrm{keV}$ annihilation line observed by SPI/INTEGRAL. Further, prompt gamma-ray emission by these decaying dark photons gives a signal for gamma-ray telescopes. Existing GRS observations of SN1987a already constrain this, and a future nearby SN could provide a detection. Finally, dark photon decays from extragalactic SN would produce a diffuse flux of gamma rays observable by detectors such as SMM and HEAO-1. Together these observations can probe dark photon couplings several orders of magnitude beyond current constraints for masses of roughly $1-100 \mathrm{MeV}$.

KeYwords: Beyond Standard Model, Cosmology of Theories beyond the SM

ArXiv EPrint: 1901.08596 


\section{Contents}

1 Introduction 1

2 Dark photon production 2

3 Positron bounds 4

3.1 Galactic positrons and supernova progenitors 4

3.2 Positron escape 5

3.3 Fireball 6

4 Gamma-ray bounds $\quad 9$

4.1 SN 1987a

$\begin{array}{lll}4.2 & \text { Diffuse extragalactic flux } & 10\end{array}$

5 Discovery potential $\quad 11$

6 Results 12

$\begin{array}{lll}7 & \text { Conclusion } & 13\end{array}$

$\begin{array}{ll}\text { A Profile dependence } & 15\end{array}$

B Dark photon decays with hard photon emission 16

\section{Introduction}

New degrees of freedom beyond the Standard Model could be hiding at energies significantly below the weak scale if the new particles have very weak couplings to the Standard Model (SM). Such scenarios are often known as "dark sectors" and naturally appear in many extensions of the SM. In particular, dark matter, which represents more than $80 \%$ of the matter density of the universe and whose identity remain one of the biggest mysteries in physics, could be part of a dark sector.

The new particles in dark sector models can only interact with the SM via a mediator. Though there is a rich experimental program searching for dark sectors and the corresponding mediators (see e.g. refs. $[1,2]$ ), if the mediators only interact very weakly with SM degrees of freedom, they could evade these searches and remain hidden. Astrophysical probes can greatly enhance the sensitivity reach for such scenarios, trading the precision associated with the controlled environment of a lab for the enormous densities and temperatures of stars [3]. 
One of the simplest and most well-motivated mediators is the dark photon, a $\mathrm{U}(1)^{\prime}$ gauge boson kinetically mixed with the SM photon [4]. In this paper, we focus on the scenario in which the dark photon decays purely into electron-positron pairs, which is the natural expectation if the dark photon is lighter than the other particles in the dark sector and is above the electron-positron mass threshold. At the energies we are interested in, the relevant terms in the Lagrangian containing the dark photon are:

$$
\mathcal{L} \supset \frac{1}{2} m^{\prime} A_{\mu}^{\prime} A^{\prime \mu}-\frac{1}{4} F_{\mu \nu}^{\prime} F^{\prime \mu \nu}-\frac{\epsilon}{2} F_{\mu \nu}^{\prime} F^{\mu \nu},
$$

where $A^{\prime}$ is the dark photon and $\epsilon$ is the mixing parameter.

A strong constraint in this model parameter space comes from supernovae cooling considerations using the "Raffelt criterion," which simply states that if any new particle could transport energy out of the SN core more efficiently than the neutrinos, it would significantly alter the cooling timescale of the proto-neutron star in conflict with existing measurements of SN 1987a [3]. Quantitatively, this amounts to asserting that if a new particle carries more than $3 \times 10^{52} \mathrm{erg} / \mathrm{s}$ out of the neutrinosphere, it is excluded. This bound was recently updated in refs. $[5,6]$ to include finite temperature and density effects in the dark photon production which lead to significant changes compared to the original calculations.

Dark photons produced within the core of a supernova can escape the progenitor star before decaying into electrons and positrons. In this work we point out that even in the regime where too few dark photons escape to yield a cooling bound, observable signatures are still produced by their decays. We show that prompt gamma-ray emission from the next galactic supernova would allow for the discovery of the dark photon. Furthermore, we use current measurements to constrain the mixing parameter in the regime where the coupling is too weak to lead to a cooling bound. We apply three different constraints to cover all of our parameter space. These are: (1) requiring that the decays of dark photons produced in supernovae do not lead to an overabundance of galactic positrons, (2) requiring that prompt gamma-ray emission from dark photon decays is not in conflict with observations of SN1987a, and (3) requiring that any plasmas formed by the decay products do not lead to an excess of extragalactic gamma rays over current measurements.

\section{Dark photon production}

In this section, we reproduce the standard computation for dark photon emission from a supernova. For a discussion of new bounds, see sections 3 through 5 .

The temperatures inside the $\mathrm{SN}$ core reach $\sim 30 \mathrm{MeV}$, hence dark photons of masses less than about $100 \mathrm{MeV}$ can be thermally produced in significant numbers within the SN. Plasma effects also cause resonant production of dark photons at sufficiently high electron densities. We follow the calculation described in ref. [5] to determine the total flux.

The differential number flux of dark photons from the SN is given by

$$
\frac{d N}{d V d t}=\int d \omega \frac{d N}{d V d t d \omega}=\int \frac{d \omega \omega^{2} v}{2 \pi^{2}} e^{-\omega / T}\left(\Gamma_{\mathrm{abs}, L}^{\prime}+2 \Gamma_{\mathrm{abs}, T}^{\prime}\right)
$$


where $T$ is the temperature, $v$ is the velocity, and $\Gamma_{\mathrm{abs}, L / T}^{\prime}$ is the absorptive width of the dark photon for the longitudinal/transverse modes. We consider only inverse bremsstrahlung, which is the dominant absorptive process inside the core. The absorptive widths are then given by

$$
\Gamma_{\mathrm{ibr}, L \mid T}^{\prime}=\frac{32}{3 \pi} \frac{\alpha\left(\epsilon_{m}\right)_{L \mid T}^{2} n_{n} n_{p}}{\omega^{3}}\left(\frac{\pi T}{m_{N}}\right)^{3 / 2}\left\langle\sigma_{n p}^{(2)}(T)\right\rangle\left(\frac{m^{\prime 2}}{\omega^{2}}\right)_{L}
$$

where $n_{n}$ and $n_{p}$ are the neutron and proton number densities respectively, $m_{N}=938 \mathrm{MeV}$, $\left\langle\sigma_{n p}^{(2)}(T)\right\rangle$ is the averaged neutron-proton dipole scattering cross-section (taken from [7]), $\left(\epsilon_{m}\right)_{L \mid T}^{2}$ is the in-medium mixing angle, and the final term is denoted with a subscript $L$ to indicate that it is only included for the longitudinal mode.

Plasma effects cause the mixing parameter $\epsilon$ to change in medium. We can find this $\left(\epsilon_{m}\right)_{L \mid T}^{2}$ by using

$$
\left(\epsilon_{m}\right)_{L \mid T}^{2}=\frac{\epsilon^{2}}{\left.\left(1-\operatorname{Re} \Pi_{L \mid T} / m^{\prime 2}\right)^{2}+\left(\operatorname{Im} \Pi / m^{\prime 2}\right)^{2}\right)}
$$

with $\Pi$ the photon polarization tensor [8]. The real part of the polarization tensor for the two modes is given by

$$
\begin{aligned}
& \operatorname{Re} \Pi_{L}=\frac{3 \omega_{p}^{2}}{v^{2}}\left(1-v^{2}\right)\left[\frac{1}{2 v} \ln \left(\frac{1+v}{1-v}\right)-1\right] \\
& \operatorname{Re} \Pi_{T}=\frac{3 \omega_{p}^{2}}{2 v^{2}}\left(1-v^{2}\right)\left[1-\frac{1-v^{2}}{2 v} \ln \left(\frac{1+v}{1-v}\right)\right]
\end{aligned}
$$

with $v=k / \omega$ and $\omega_{p}$ the plasma frequency, which for a gas of degenerate electrons is given by

$$
\omega_{p}^{2}=\frac{4 \pi \alpha_{\mathrm{EM}} n_{e}}{\sqrt{m_{e}^{2}+\left(3 \pi^{2} n_{e}\right)^{2 / 3}}}
$$

where $n_{e}$ denotes the number density of electrons [9].

Within the SN, the SM photons are in thermal equilibrium. Hence the imaginary part of the polarization tensor just becomes

$$
\operatorname{Im} \Pi_{L, T}=-\omega\left(1-e^{-\omega / T}\right) \Gamma_{\mathrm{abs} \mid L, T}
$$

where $\Gamma_{\mathrm{abs} \mid L, T}$ is the absorptive width of the Standard Model photon, taken to be

$$
\Gamma_{\mathrm{ibr}, L \mid T}=\frac{32 \alpha}{3 \pi} \frac{n_{n} n_{p}}{\omega^{3}}\left(\frac{\pi T}{m_{N}}\right)^{3 / 2}\left\langle\sigma_{n p}^{(2)}(T)\right\rangle\left(\frac{m^{\prime 2}}{\omega^{2}}\right)_{L} .
$$

In other words, $\Gamma_{\text {abs }}^{\prime}=\left(\epsilon_{m}\right)_{L \mid T}^{2} \Gamma_{\text {abs }}$.

By using all of the above relations, we can now determine $d N / d V$ as a function of radius. The radial dependence comes in the form of the radially-dependent parameters $n_{n}(r), n_{p}(r), n_{e}(r)$, and $T(r)$. We use the same fiducial profile as ref. [5], which has the 
functional form

$$
\begin{array}{r}
\rho(r)=\rho_{c} \times \begin{cases}1+k_{\rho}\left(1-r / R_{c}\right) & r<R_{c} \\
\left(r / R_{c}\right)^{-\nu} & r \geq R_{c}\end{cases} \\
T(r)=T_{c} \times \begin{cases}1+k_{T}\left(1-r / R_{c}\right) & r<R_{c} \\
\left(r / R_{c}\right)^{-\nu / 3} & r \geq R_{c}\end{cases}
\end{array}
$$

with $k_{\rho}=0.2, k_{T}=-0.5, \nu=5, R_{c}=10 \mathrm{~km}, \rho_{c}=3 \times 10^{14} \mathrm{~g} / \mathrm{cm}^{3}, T_{c}=30 \mathrm{MeV}$, and constant $Y=0.3$. We have chosen to use this profile since we find that it produces slightly more conservative bounds in comparison to other profiles, though we find that the bounds change little even under a large variation in the profile. (See appendix A.)

We perform the integral $\int \frac{d N}{d t d V} d V$ over these radial profiles to compute the total dark photon flux. This flux is dominated by regions within or near the core, so the upper limit of this integral can be taken to be $\sim 50 \mathrm{~km}$ without affecting the total flux. In the region of parameter space we are focusing on, the mean free path of the dark photon is sufficiently large such that we can safely neglect possible reabsorption near the core. ${ }^{1}$

\section{Positron bounds}

In this section, we show that positrons can provide a direct signature of dark photons emitted by supernovae and discuss their behavior in a variety of regimes.

\subsection{Galactic positrons and supernova progenitors}

The results from the SPI gamma ray spectrometer on the INTEGRAL satellite have greatly improved the measurements of the galactic $511 \mathrm{keV}$ gamma ray flux [10], which in turn have allowed for more accurate estimates of the galactic positron annihilation rate. Estimates of this rate vary depending on the particular galactic model used, but suggest a rate no larger than $\sim 4 \times 10^{43} \mathrm{~s}^{-1}$ [11]. Assuming that positron production and annihilation are in equilibrium, this can be taken as the galactic positron production rate as well. If some set of astrophysical sources were to produce positrons in excess of this rate, the resulting positrons would produce an excess of $511 \mathrm{keV}$ photons in the galaxy in disagreement with INTEGRAL's result. Using only the bound from the $511 \mathrm{keV}$ gamma ray flux to constrain new positron sources sets a conservative bound. A more detailed analysis of positron injection in the galaxy with energies in the $10-10^{3} \mathrm{MeV}$ range would conceivably lead to even stronger constraints $[12,13]$, but it is beyond the scope of this paper.

We focus on positron production in two classes of SN: type II and Type Ib/c. While both are core-collapse SN, they differ critically in the size of the progenitor star and the density of gas outside the photosphere, both of which play important roles in determining the overall positron flux that escapes the SN. Note however that the collapse of the core

\footnotetext{
${ }^{1}$ It should be noted that the density profile in eq. (2.9) is only applicable near the core, as one would expect an exponential decrease in density at large radii. However, since in the regions of parameter space of interest, the dark photons are produced in the core and escape without interacting with the SN overburden, the dependence at large radii does not affect the analysis.
} 
and formation of the proto-neutron star are unaffected by the outer layers of the star, hence both Type II and Ib/c SN are well-modeled by the above profile.

The progenitors of Type II SN, specifically the more common Type IIp variety, are thought to be almost exclusively red supergiants (RSGs) [14]. Red supergiants can have stellar radii of over $1400 R_{\odot}$, or roughly $10^{9} \mathrm{~km}$. Above the star's photosphere, there is a gaseous atmosphere that decreases in molecular number density from a maximum of $\sim 10^{10} \mathrm{~cm}^{-3}$ at $10^{9} \mathrm{~km}$ in an approximately $r^{-2}$ fashion [15]. Type II SN occur in our galaxy with an average rate of $2 \mathrm{SN} /$ century [16]. Therefore, if Type II SN were to contribute $6.3 \times 10^{52}$ positrons per $\mathrm{SN}$, this would saturate the observed galactic positron flux. With this in mind, we take the criterion that any dark photon that allows Type II SN to produce $>10^{53}$ positrons is excluded.

Type Ib/c are similar to Type II SN in that they are core-collapse SN, but they typically occur in hydrogen-stripped stars. Their progenitors are far smaller than RSGs, typically having radii $\lesssim 10 R_{\odot}$ [17], and have already shed their outermost layers of hydrogen (and helium, for Type Ic), either due to stripping by a companion star or through stellar winds.

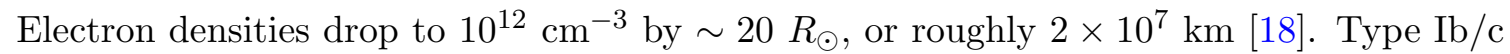
are about an order of magnitude less frequent than Type II SN in our galaxy [19], so for our condition on Type Ib/c SN, we take that any dark photon that allows Type Ib/c SN to produce $>10^{54}$ positrons is excluded.

\subsection{Positron escape}

It is imperative for this bound to apply that the dark photons escape the progenitor star before decaying, otherwise the resulting positrons are likely to annihilate unobserved within the star's outer layers and gaseous envelope. If the positrons are able to escape the star, they have a long lifetime $\left(10^{5}\right.$ to $10^{6}$ years $)$ in the interstellar medium and can slow down and contribute to the observable $511 \mathrm{keV}$ line [20].

We can estimate the radius $r_{\text {esc }}$ at which an emitted positron would have an order one survival probability using the standard formula for positron annihilation rate in a gas. A conservative choice is to take this to be $\Gamma_{e^{+}}=\pi r_{0}^{2} n_{e}$ [21], where $r_{0}$ is the 'classical electron radius' $\left(2.8 \times 10^{-13} \mathrm{~cm}\right)$ and $n_{e}$ is the electron density, hence we have an interaction length of $\lambda \sim\left(\pi r_{0}^{2} n_{e}\right)^{-1}$. In a Type Ib/c progenitor, $\lambda \sim r$ at roughly $20 R_{\odot}$, so at radii larger than this, most positrons produced in the decay of the dark photon can escape without annihilation with electrons in the star's outer layers. We therefore set $r_{\text {esc }}=2 \times 10^{7} \mathrm{~km}$ for Type Ib/c. By the same argument, we find $r_{\text {esc }}=10^{9} \mathrm{~km}$ for a Type II SN.

Note that since the SN shock begins well within the star, the external layers of the progenitor star do not feel the effects of the SN until the shock has propagated out to them. The shock speed can get up to a third of the speed of light [22], so in the case of a Type $\mathrm{Ib} / \mathrm{c} \mathrm{SN}$, it takes roughly $10^{2}$ seconds for the region at which an emitted positron would escape to feel the effects of the collapse. For this reason, we can treat the outer layers of the star as having no knowledge of the shock when computing the escape probabilities on the timescale of dark photon emission ( $\equiv \Delta t \sim 10$ seconds).

Therefore, for Type Ib/c (Type II) SN we must have the total dark photon flux through a radius $r_{\text {esc }}=2 \times 10^{7} \mathrm{~km}\left(10^{9} \mathrm{~km}\right)$ be greater than $10^{54}\left(10^{53}\right)$ in order to exclude that 
part of parameter space. The decay width of the dark photon at rest to $e^{+} e^{-}$in vacuum is given by the following expression:

$$
\Gamma=\frac{1}{3} \alpha_{\mathrm{EM}} \epsilon^{2} m^{\prime} \sqrt{1-\frac{4 m_{e}^{2}}{m^{\prime 2}}}\left(1+\frac{2 m_{e}^{2}}{m^{\prime 2}}\right)
$$

It is appropriate to use the vacuum decay width as opposed to the plasma decay width because for the mixing parameters we are considering the dark photons decay length is much larger than the core radius, which is where the finite temperature and density effects are relevant. The decay length is then $d=\frac{\omega v}{m^{\prime} \Gamma}$ with $\omega$ the dark photon energy, $v$ the velocity, and $\Gamma$ the decay width in its rest frame.

Using this, our constraint for Type Ib/c SN takes the form

$$
\Delta t \int d V d \omega \frac{d N}{d V d \omega d t} e^{-\left(2 \times 10^{7} \mathrm{~km}\right) / d}>10^{54} \text { positrons }
$$

and the equivalent for Type II SN is

$$
\Delta t \int d V d \omega \frac{d N}{d V d \omega d t} e^{-\left(10^{9} \mathrm{~km}\right) / d}>10^{53} \text { positrons. }
$$

Since which of these two conditions provides a stronger bound differs over parameter space, it is more convenient to combine them into a single condition that takes into consideration both contributions from galactic Type II and Type Ib/c SN:

$$
\Delta t \int d V d \omega \frac{d N}{d V d \omega d t}\left(e^{-\left(10^{9} \mathrm{~km}\right) / d}+0.1 e^{-\left(2 \times 10^{7} \mathrm{~km}\right) / d}\right)>10^{53} \text { positrons. }
$$

This is the constraint we use to generate our bounds.

\subsection{Fireball}

So far we have focused on the effects of the outer layers of the progenitor star on the positron propagation. Another important effect is the interaction of the positrons with the electrons created in the dark photon decay. As pointed out in ref. [23], if a large number of dark photons decay in a shell just outside the photosphere, they can create an opticallythick plasma of electrons, positrons, and photons. Unfortunately, their analysis neglected two important features: the thermal effects on the production of the dark photon (which are large for lighter dark photons) and a detailed analysis of the emission timescale and spectrum of the photons produced, which require more detailed modeling of the plasma. This can be seen clearly, for example, by the fact that the bounds placed in ref. [23] are independent of mass at low coupling, which is the result of neglecting thermal effects. As a result, the bounds presented in ref. [23] are incorrect and should be replaced by the bounds presented in this paper.

The dynamics of this plasma resembles that of the fireball model [24, 25] which is used to describe Gamma-Ray Bursts (GRBs). However, it differs from the usual fireball models in an important aspect: the energy density in the plasma is significantly smaller than the one considered for GRBs. To emphasize this difference we will henceforth refer to 
this plasma as a dilute fireball. In these dilute fireballs, both the initial number densities and the temperature are related to the original dark photon number density and mass. Although the dark photons will decay through a large volume, we will focus on a spherical shell of width $d$ and radius $R_{*}=\min \left(d, r_{\text {esc }}\right)$, where $d$ is the average decay length of the dark photons and $r_{\text {esc }}$ is the escape radius discussed in the previous section. An order one fraction of the dark photons that decay outside the star will decay in this shell.

As in the fireball model, it is easiest to understand local properties of the plasma by boosting to a frame that is moving with the plasma, i.e. in which the momentum distribution of the particles in the plasma is isotropic. If the original dark photon flux was monochromatic this frame would coincide with the dark photons' rest frame, and the boost factor would be $\eta=E_{\gamma^{\prime}} / m^{\prime}$. In our analysis we will neglect the spread in dark photons' initial momenta, which is justified if the velocity spread of the dark photons does not lead to a spatial spread of the dark photon flux that is larger than the average dark photon decay length. Because we are interested in dark photons with lifetimes $\tau \gg 10$ seconds, we can neglect the initial time spread of the dark photons and estimate the initial electron/positron density in the comoving frame as

$$
n_{e}\left(R_{*}\right) \sim \frac{\Delta N_{\gamma^{\prime}}}{4 \pi R_{*}^{2}(d / \eta)}
$$

where $\Delta N_{\gamma^{\prime}}$ is the number of dark photons that decay inside the shell and $(d / \eta)$ is the Lorentz-contracted width of the shell. The internal energy of the leptons is determined by the mass of the dark photon, and therefore if they form a plasma, the initial temperature is $T \sim m^{\prime}$.

One can easily check that the initial number density of the plasma is significantly below the thermal number density of a plasma with the same temperature $\left(\sim m^{\prime 3}\right)$. This leads to a much smaller optical depth than in the typical fireball model. The initial optical depth for the shell is well-approximated as

$$
\tau_{0} \approx n_{e} \sigma_{e^{+} e^{-} \rightarrow \gamma \gamma}(d / \eta) \sim\left(\frac{\Delta N_{\gamma^{\prime}}}{4 \pi R_{*}^{2}(d / \eta)}\right)\left(4 \pi \frac{\alpha^{2}}{T^{2}}\right)(d / \eta) \sim\left(\frac{\alpha^{2} \Delta N_{\gamma^{\prime}}}{m^{\prime 2} R_{*}^{2}}\right)
$$

If it is larger than one, a plasma forms and the reaction $e^{+} e^{-} \leftrightarrow \gamma \gamma$ is in local equilibrium and the photon and lepton number densities are related by detailed balance. ${ }^{2}$

The initial temperature of the plasma is at least as large as the dark photon mass, and hence initially the total number densities of positrons and photons are comparable. The only way to have a significant decrease in the number of positrons is if there is an efficient way to cool the plasma so that the temperature becomes smaller than the electron mass

\footnotetext{
${ }^{2}$ In the presence of large magnetic fields the optical depth could be enhanced due to charged particles being trapped by the fields. However one can easily show that the kinetic energy in the plasma is orders of magnitude larger than the expected energy density in magnetic fields in the region of interest. Therefore, the magnetic field would be combed out by the plasma and would not lead to efficient trapping. (For details on radially-combed magnetic fields, see e.g. [20].)
} 
$m_{e}$. There are two important ways in which the plasma can cool: internal processes and adiabatic expansion. ${ }^{3}$

Internal processes are important if reactions that can change the total number of particles, in particular 2-to-3 reactions (e.g. $e^{+} e^{-} \rightarrow 3 \gamma$ ), are occurring in the plasma. Such processes increase the number density, driving it towards $T^{3}$, and consequently lower the temperature due to energy conservation. The optical depth for such reactions is approximately a factor of $\alpha$ lower than the optical depth for the 2-to-2 process. In figure 1, we indicate the region in which the 2-to-3 optical depth is larger than 0.1 with a red dashed line. In this region, we expect that a large fraction of positrons annihilate and therefore cannot place a positron injection bound there. However, we can place a bound on this region using the resulting gamma rays (see section 4.2).

Even if 2-to-3 processes are off, if 2-to-2 processes are on, the plasma can still cool due to adiabatic expansion, as occurs in a thin region of our parameter space. Unlike in the situation when 2-to-3 processes are on, we can still place a positron injection bound in this region. The cooling due to the expansion is analogous to the case of the regular fireball (see refs. [24, 25] for a detailed discussion). As the plasma expands it converts the internal gas energy (temperature) to kinetic energy of the expanding shell, i.e. as the plasma expands, most of the momentum becomes radial. For a plasma with energy density dominated by radiation, this leads to the scaling $T \propto 1 / R$, where $R$ is the radius of the expanding shell in the original star frame. As a result, when $T>m_{e}$, the cross-section scales as $\sigma \propto 1 / E_{\mathrm{CM}}^{2} \propto 1 / T^{2} \propto R^{2}$ and the number density scales as $n_{e}(R) \propto 1 / R^{2}$ (where we ignore the growth of the shell width as it cancels in the optical depth). Therefore, one can easily see that the optical depth remains constant while $T>m_{e}$.

Eventually, at some radius $R_{e}$, the temperature will drop below the electron mass, causing the number density and cross-section to begin scaling differently. The number density will scale as

$$
n_{e}(R) \approx n_{e}\left(R_{e}\right)\left[\left(\frac{R_{e}}{R}\right)^{2}\left(\frac{m_{e}}{T}\right)^{3 / 2} e^{-m_{e} / T}\right]
$$

due to Boltzmann suppression and the cross-section begins to scale as $\sigma \propto 1 /\left(E_{\mathrm{CM}}^{2} v\right) \propto$ $T^{-1 / 2} \propto R^{1 / 2}$ since $E_{\mathrm{CM}}$ is fixed at roughly $m_{e}$ but the velocity $v \sim \sqrt{T / m_{e}}$. Therefore, we see immediately that the $R$-dependence cancels in the optical depth except for an exponential factor and $\tau$ scales as $\tau \propto \exp \left(-R / R_{e}\right)$.

In the regions where 2 -to- 2 reactions are occurring but 2 -to- 3 are not, we necessarily have that the 2 -to- 2 optical depth is $1 \lesssim \tau \lesssim 10$. Therefore, to decrease this optical depth below one such that positrons can escape, we see that we must only expand by at most $\sim 3 R_{e}$. Using this and eq. (3.7), we see that the total number of positrons escaping the supernova is only an order-one factor smaller than the initial number produced at $R_{*}$, hence the formation of a fireball has little ultimate effect on the flux when it is sufficiently dilute

\footnotetext{
${ }^{3}$ Large baryon loading could also in principle lead to significant cooling. However, since we are considering decays in regions where the stellar atmosphere is very diffuse, one can show that the number density of baryons is too small to lead to appreciable cooling.
} 
such that number-changing processes are not occurring. We include this small suppression when computing the bounds in figure 1.

\section{Gamma-ray bounds}

In this section, we introduce two distinct gamma-ray signatures of SN-produced dark photons that we then use to constrain new regions of dark photon parameter space.

\subsection{SN 1987a}

There is an additional signal that can be used to constrain dark photons in this parameter space, namely the absence of an observed gamma-ray flux above background in the first few minutes following the arrival of SN1987a's neutrinos on Earth.

The non-observation of an increased gamma-ray flux by the Gamma Ray Spectrometer (GRS) aboard the Solar Maximum Mission results in the following fluence limits for the first 270 seconds, taken from ref. [26]: $f_{\gamma} \lesssim 5 \mathrm{~cm}^{-2}(4-6 \mathrm{MeV}) ; f_{\gamma} \lesssim 1.6 \mathrm{~cm}^{-2}(10-25 \mathrm{MeV})$; and $f_{\gamma} \lesssim 3.3 \mathrm{~cm}^{-2}(25-100 \mathrm{MeV})$. Since the exact spectrum of the outgoing gamma rays depends on the choice of profile, we choose to place a conservative limit by summing the fluences in all bins and requiring that the total number of gamma rays produced does not exceed it. This results in a fluence limit of $f_{\gamma} \lesssim 10 \mathrm{~cm}^{-2}$, which translates to a limit on the number of hard gamma rays escaping the supernova of $N_{\gamma}<4 \times 10^{48}$.

In order to use the quoted fluence limit, we must require that the arrival time of the resulting gamma rays on Earth be less than 270 seconds after the arrival time of the neutrinos. The massive dark photons are not traveling exactly at the speed of light, so before they decay, they accumulate some time delay behind the neutrinos. We therefore compute a minimum boost required such that the dark photons reach the distance at which the gamma rays are primarily produced before this delay reaches 270 seconds. This distance is taken to be $R_{*}=\max \left(r_{1987}, d\right)$, with $d$ the decay length of the dark photon and $r_{1987}$ the escape radius for SN1987a's progenitor $\left(r_{1987}=4 \times 10^{7} \mathrm{~km}[27]\right)$ since if $d>r_{1987}$, then the majority of decays will occur by $r=d$.

We consider this bound only in the regime where a plasma never forms since, as argued above, it is likely that a fireball would result in gamma rays below the GRS lower limit of $4 \mathrm{MeV}$. In this region of parameter space, hard gamma rays can be produced as final state radiation with a rate that is suppressed by roughly $\alpha$ with respect to the exclusive $e^{+} e^{-}$ decays. Therefore the number of these hard gamma rays produced is

$$
(\Delta t) \int d V \int_{\gamma_{\min }}^{\infty} d \omega \frac{d N}{d V d t d \omega}\left(e^{-r_{1987} / d} \operatorname{Br}\left(e^{+} e^{-} \gamma_{>4 \mathrm{MeV}}\right)\right)
$$

where $\Delta t=10$ seconds is the emission timescale, $\gamma_{\min }$ is the minimum boost required such that the decays occur within 270 seconds, and $\operatorname{Br}\left(e^{+} e^{-} \gamma_{>4 \mathrm{MeV}}\right)$ is the branching ratio for a dark photon with energy $\omega$ to decay to a photon with energy above $4 \mathrm{MeV}$. (See appendix $\mathrm{B}$ for a derivation of this branching ratio.) If this quantity ever exceeds $4 \times 10^{48}$ gamma rays, we place a constraint on the corresponding region of parameter space. This effect also has discovery potential: if we were to measure the spectrum of the next galactic SN 
and find an anomalous gamma-ray excess, it could provide evidence for dark photons. (See section 5.)

Ref. [23] attempts to use the same GRS non-observation to place bounds on dark photons using gamma rays produced in a fireball, but neglects to compute the outgoing spectrum of the resultant gamma rays, which are expected to have energies $<4 \mathrm{MeV}$. There does not appear to be any data for these lower energy bins, hence a bound cannot be placed by GRS in this regime.

\subsection{Diffuse extragalactic flux}

The only remaining region of parameter space left to constrain is the region in which a fireball forms and 2-to-3 processes are occurring. This can be partially accomplished by comparing the diffuse extragalactic flux of gamma-rays that would be generated by these fireballs to the measured value.

It is well-known that extragalactic supernovae lead to a diffuse background of supernova neutrinos. In the same fashion, the collective effect of extragalactic supernovae producing these 2-to-3 fireballs would be to contribute to the diffuse extragalactic background of gamma rays. We can easily compute the diffuse energy flux in a given energy bin on Earth by performing a line-of-sight integral over distance in the following way [28]:

$$
\Phi_{\gamma}=\int_{0}^{\infty} E_{\gamma}^{\text {bin }} R_{S N}(z)\left|\frac{d t}{d z}\right| d z
$$

where $E_{\gamma}^{\text {bin }}$ is the total energy of gamma-rays in the specified bin produced by a single $\mathrm{SN}, R_{S N}(z)$ is the redshift-dependent supernova rate (taken from [28]), and $\left(\frac{d t}{d z}\right)^{-1}=$ $H_{0}(1+z) \sqrt{\Omega_{\Lambda}+\Omega_{m}(1+z)^{3}}$.

We restrict our attention to gamma rays with energy between $100 \mathrm{keV}$ and $4 \mathrm{MeV}$. This is simply due to the fact that for energy greater than $4 \mathrm{MeV}$, the GRS bound would apply and by $T \sim 100 \mathrm{keV}$, the fireball has become transparent to gamma-rays. The extragalactic flux in this bin has been measured by the Solar Maximum Mission (SMM) [29] and the High Energy Astronomy Observatory (HEAO-1) [30], which allows us to integrate over this bin to find a total flux on Earth in this energy range. Performing the integral, we get a value of roughly $0.5 \mathrm{MeV} \mathrm{cm}{ }^{-2} \mathrm{~s}^{-1}$. It is therefore simple to determine what total energy released by a single supernova would lead to an excess over the measured extragalactic flux. This calculation yields a bound of $\sim 1.2 \times 10^{56} \mathrm{MeV}$ in gamma rays with $0.1 \mathrm{MeV}<E_{\gamma}<4 \mathrm{MeV}$ for Type II SN and $\sim 1.2 \times 10^{57} \mathrm{MeV}$ for a Type Ib/c. These bounds are conservative in that they assume the spectrum of gamma rays has the same shape as the background. A more careful analysis of the spectrum would improve these bounds.

The total initial energy of the dark photons that escape the star has to be released from the supernova in some form. If even a small fraction of it were released as positrons, we would be able to place a positron bound on the 2-to-3 fireball region. Hence we assume here that all the energy is released in the form of gamma rays, which allows us to simply compare the total energy of the dark photons that decay outside of $r_{\mathrm{esc}}=2 \times 10^{7} \mathrm{~km}$ to the 
extragalactic bound on energy we have just computed. ${ }^{4}$ We find that this simple argument covers a band of parameter space within the 2-to-3 fireball region ("Gamma rays (diffuse flux)" in figure 1), though does not constrain the entirety of the region. Regions in which this fails to place a constraint are colored in yellow. A careful analysis of the spectrum of gamma rays emitted by the expanding fireball would likely constrain these regions as well, but it is beyond the scope of this paper.

\section{Discovery potential}

In this section, we explain how a future observation of the next galactic SN could allow us to discover the dark photon through gamma rays produced in its decays.

While the previous sections have addressed bounds we can place on dark photons using existing observations, we can additionally consider the case of a future nearby supernova and ask in what regions of parameter space we would have sensitivity to discovering the dark photon. With a galactic SN rate of $\sim 2$ per century, it is not unreasonable to expect to observe one in the next few decades. With this in mind, we seek to compute a rough estimate of the parameter space we could probe with such a future observation.

The signature we will be searching for is the same as for the SN1987a bound, namely the gamma-ray flux from hard photons produced in three-body decays of the dark photon. We take as our background a conservative estimate of the diffuse gamma-ray flux from the galactic center in the energy band $1-100 \mathrm{MeV}$, as this exceeds the expected gamma-ray flux from the SN itself in the absence of BSM processes. As measured by COMPTEL [31], a gamma-ray telescope aboard the NASA Gamma Ray Observatory, the background for this bin can be estimated as $E^{2} \frac{d \Phi}{d E} \lesssim 0.01 \mathrm{MeV} \mathrm{cm}{ }^{-2} \mathrm{~s}^{-1} \mathrm{sr}^{-1}$. Taking $d=8 \mathrm{kpc}$ (the distance from Earth to the center of the galaxy), this becomes a rate of $(d N / d t)_{\gamma} \sim 10^{44} \mathrm{~s}^{-1}$. Note that this is not a true production rate of gamma rays by the $\mathrm{SN}$, but is rather the total number of gamma rays ultimately produced divided by the time interval over which they arrive at Earth.

In order to determine the dark photon production required to exceed this gamma-ray flux, consider a shell of dark photons emitted from the SN. As dark photons decay, the associated gamma rays escape at $c$ while the remaining dark photons lag behind. Once an $\mathcal{O}(1)$ fraction of the dark photons have decayed (after roughly $\gamma_{\text {avg }} \tau$, their Lorentz-dilated lifetime), the gamma-rays will have a time-spread of roughly $(1-v) \gamma_{\text {avg }} \tau$, where $v$ is the average velocity of the dark photons and $\gamma_{\text {avg }}$ is the average boost of the dark photons. Therefore, to find a decay rate, we simply divide the total dark photon production by this time-spread (and multiply by the fraction of decays that will produce a gamma ray with energy $>1 \mathrm{MeV}, \operatorname{Br}\left(e^{+} e^{-} \gamma_{>1 \mathrm{MeV}}\right)$, the derivation of which appears in appendix $\left.\mathrm{B}\right)$. Hence we have the relation

$$
(d N / d t)_{\gamma}=\operatorname{Br}\left(e^{+} e^{-} \gamma_{>1 \mathrm{MeV}}\right) N_{A^{\prime}} \min \left[\left(\frac{1}{1-v}\right) \frac{1}{\gamma_{\text {avg }} \tau}, \frac{1}{10 \mathrm{sec}}\right]
$$

\footnotetext{
${ }^{4}$ We focus purely on Type Ib/c SN since in this region of parameter space, the dark photon decay length is significantly less than the radius of a Type II progenitor, hence the escaping flux from a Type II SN suffers from a large exponential suppression.
} 
The minimum function appears only because the time-spread can never be smaller than the initial 10-second emission timescale of the dark photons. Note that $N_{A^{\prime}}$ is the total number of dark photons that will escape the SN and is simply given by the expression on the left-hand side of eq. (3.2) for Type Ib/c and eq. (3.3) for Type II. Using our condition that $(d N / d t)_{\gamma}>10^{44} \mathrm{~s}^{-1}$, we can estimate the sensitivity limits.

Since it is roughly an order of magnitude more likely that the next supernova we observe will be a Type II SN, we have indicated those bounds in solid blue in figure 1 . If it were to be a Type Ib/c, however, the smaller escape radius would allow us to be sensitive to an even larger region of parameter space, which we have chosen to indicate with a dotted blue line in figure 1. Recall that an assumption used to compute these bounds is that no fireball forms, hence they do not extend into the unconstrained yellow regions in figure 1. However, observations of the $\sim 100 \mathrm{keV}$ gammas produced in the fireball of a nearby SN would likely constrain these regions as well.

Though it may seem surprising that the Type II discovery region does not enclose the SN1987a gamma-ray bound, this is due entirely to the fact that SN1987a's escape radius was $\sim 4 \times 10^{7} \mathrm{~km}$, which is two orders of magnitude smaller than the conservative $10^{9} \mathrm{~km}$ we use for a generic Type II progenitor. It is clear that the Type Ib/c discovery region (with $r_{\mathrm{esc}}=2 \times 10^{7} \mathrm{~km}$ ) fully encloses the SN1987a bound.

\section{Results}

For any given $m^{\prime}$ in our range of interest, we can easily invert our criteria to determine at what $\epsilon$ the positron or gamma-ray flux becomes too large. This places bounds on regions of parameter space up to two orders of magnitude below current bounds on dark photons from cooling constraints. The result is shown in figure 1 . The bound is cutoff on the lower end of $m^{\prime}$ by the fact that the dark photon must have $m^{\prime}>2 m_{e}$ in order for the decay to $e^{+} e^{-}$to take place.

The upper bound corresponds to where the diminishing decay length $\left(d \propto \epsilon^{-2}\right)$ results in too few dark photons escaping out of the star before decay (for larger $\epsilon$ it is possible that the energy carried by the dark photon and deposited at the outer layers of the star would lead to an early explosion which could serve as a further constraint in this region of parameter space [23]). The lower bound corresponds to where the mixing becomes too small to produce enough dark photons to violate our conditions. Note that contributions to the lower positron bound are dominated by Type II SN since at mixings this low, the exponential suppression due to decay length is not a large effect and Type II SN are an order of magnitude more common than Type Ib/c. The upper positron bound is dominated by contributions from Type Ib/c since at these higher couplings, the decay length becomes much shorter so a smaller escape radius compensates for the order of magnitude loss in abundance in comparison to Type II. The 3-body gamma-ray bound at higher masses uses SN1987a throughout. This gamma-ray bound extends to higher masses than the positron bound because it requires several orders of magnitude fewer decay products than does the positron bound. Despite this, it does not place stronger constraints on low $\epsilon$ than 
the positrons below $20 \mathrm{MeV}$ due to the increasing decay lengths resulting in fewer decays within the 270 -second window.

The region within the red dashed line denoted "Gamma rays (diffuse flux)" is the region in which number-changing processes may be occurring in the resulting electron-positron plasma outside the photosphere. Here, we use our bound from the diffuse extragalactic gamma-ray flux. The yellow regions indicate where a number-changing fireball forms but the diffuse bound fails to constrain the parameter space. The fireball no longer forms above the upper red dashed line due to too few dark photons escaping. Note that the sharp features in the upper yellow region are a numerical artifact from computing with a finite grid in parameter space and are not physical. Between the orange and red dashed lines is where only 2-to-2 process are occurring, so we have a plasma but can still place a positron injection bound (see section 3.3). Positron bounds end at the green dashed line, beyond which we can constrain parameter space using our bound from observations of SN1987a.

The blue curves in the figure indicate regions in which we would be able to observe the gamma-ray flux associated with dark photon decays if a supernova were to occur near the galactic center. The solid blue line shows the region for a Type II SN while the dotted blue line shows the region for a Type Ib/c. The Type II region does reach to as strong a coupling as the Type $\mathrm{Ib} / \mathrm{c}$ region because of the exponential suppression of the escaping flux due to the dramatically larger escape radius for Type II progenitors. However, since Type II $\mathrm{SN}$ are an order of magnitude more abundant than Type Ib/c, it is unlikely that the next galactic supernova will be a Type Ib/c. The curve is drawn simply for completeness.

\section{Conclusion}

We have used direct signals to place new bounds on models of kinetically-mixed dark photons by considering various effects of the electromagnetic decays of dark photons produced in supernovae. We have shown that if any novel particle produced within a Type II supernova produced in excess of $10^{53}$ escaping positrons (or $10^{54}$ in a Type $\mathrm{Ib} / \mathrm{c}$ ), it would result in a $511 \mathrm{keV}$ galactic emission in conflict with current observations by SPI/INTEGRAL. Additionally, we used the result that dark photons could not lead to an emission of greater than $4 \times 10^{48}$ gamma rays with $E_{\gamma}>4 \mathrm{MeV}$ in the 270 -second window following the arrival of SN1987a's neutrinos without being excluded by observations by GRS. Finally, we used SMM and HEAO-1's observations of the diffuse extragalactic gamma-ray flux to constrain the dark photons produced in any given Type Ib/c supernova from releasing more than $\sim 1.2 \times 10^{57} \mathrm{MeV}$ in gamma rays with $0.1 \mathrm{MeV}<E_{\gamma}<4 \mathrm{MeV}$. By combining these constraints, we are able to exclude a large region of parameter space two orders of magnitude weaker in coupling than current cooling bounds. Excitingly, we have also shown the potential for a discovery of the dark photon by observing the gamma ray spectrum of the next galactic supernova. These conclusions apply to any model in which the dark photon has an $\mathcal{O}(1)$ branching ratio to $e^{+} e^{-}$. Our results are summarized in figure 1. 


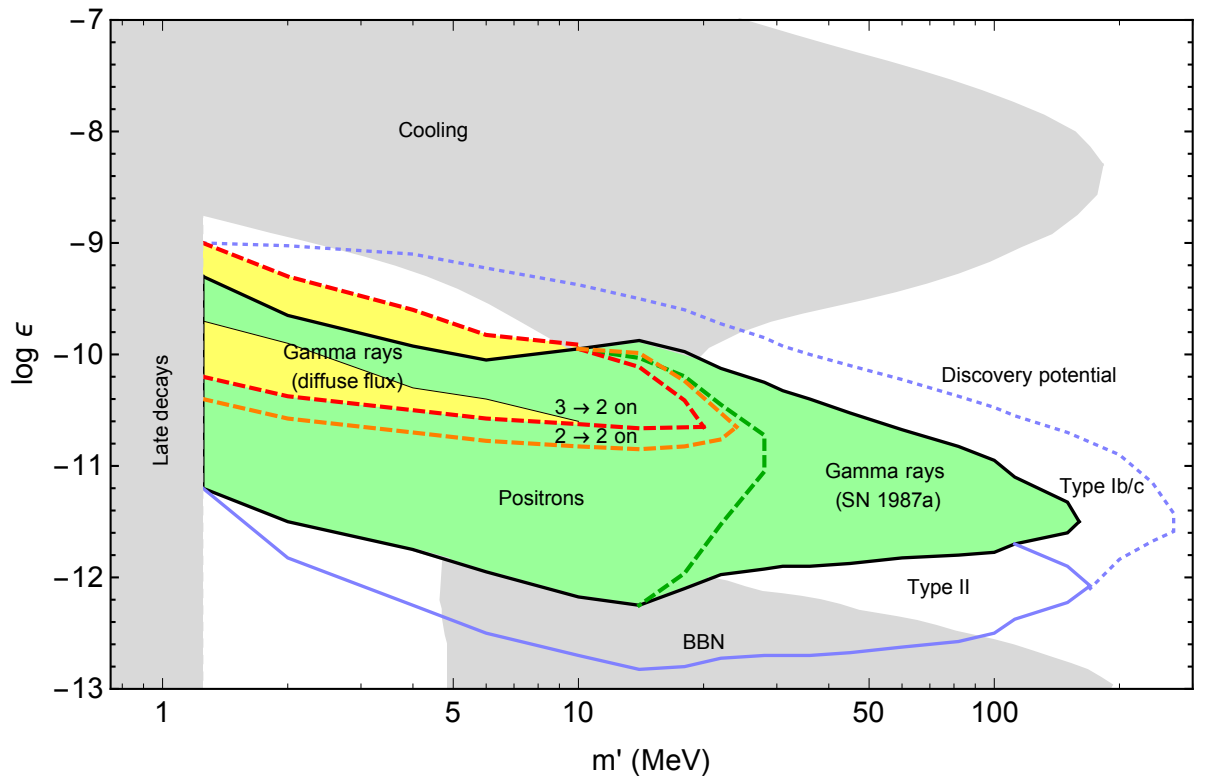

Figure 1. The overall region excluded by this work is colored in green. The blue curves show the region in which we could discover the dark photon during the next galactic SN. The solid blue curve is drawn considering the case of a Type II SN, the dotted blue curve a Type Ib/c. Yellow regions indicate where a simple analysis of the diffuse flux fails to place a constraint, though a more involved analysis would likely constrain these regions as well. The positron bound rules out parameter space between the red and the green dashed lines. The gamma-ray constraint from SN1987a extends the excluded region to higher masses (past the green dashed line), and the diffuse gamma-ray flux to stronger couplings (above the red dashed line). The orange dashed line indicates where a plasma forms but only 2-to-2 processes are on, whereas the red dashed line indicates where a fireball with 2-to-3 processes occurring forms. Previous bounds are shown in gray. The cooling bound is reproduced from [5] and the BBN constraint from [32]. The late decay bound is taken from [33] and comes from considering the decay of dark photons on cosmological timescales (see also [34]).

\section{Acknowledgments}

The authors thank Ed Hardy, Robert Lasenby, and Sam McDermott for useful discussions. W.D., G.M.T., and P.W.G. are supported by DOE Grant DE-SC0012012. W.D. and P.W.G are further supported by NSF Grant PHY-1720397, the Heising-Simons Foundation Grants 2015-037 and 2018-0765, DOE HEP QuantISED award \#100495, and the Gordon and Betty Moore Foundation Grant GBMF7946. The work of G.M.T. was also supported by the NSF Grant PHY-1620074 and by the Maryland Center for Fundamental Physics. S.R. is supported in part by the NSF under grants PHY-1638509 and PHY-1507160, the Simons Foundation Award 378243, and the Heising-Simons Foundation Grant 2015-038. D.K. is supported in part by the U.S. Department of Energy, Office of Science, Office of Nuclear Physics, under contract number DE-AC02-05CH11231 and DE-SC0017616, by a SciDAC award DE-SC0018297, by the National Science Foundation Grant PHY-1630782, and by the Heising-Simons Foundation Grant 2017-228. 


\section{A Profile dependence}

In order to estimate the uncertainty on our results due to our choice of SN profile, we compared to a profile with significant differences in both dependence and overall magnitude. The profile chosen as a comparison is given by the following.

$$
\begin{gathered}
\rho(r)=\rho_{0} \times \begin{cases}e^{-2\left(r-R_{0}\right) / R_{0}} & r<R_{0} \\
e^{\left(R_{0}-r\right) / h} & R_{0} \leq r<R_{t} \\
e^{\left(R_{0}-R_{t}\right) / h}\left(r / R_{t}\right)^{-3} & r \geq R_{t}\end{cases} \\
T(r)= \begin{cases}T_{\text {in }}+\left(T_{0} \frac{R_{0}}{R_{\text {in }}}-T_{\text {in }}\right) \exp \left[-16 \frac{\left(r-R_{\text {in }}\right)^{2}}{R_{\text {in }}^{2}}\right] & r<R_{\text {in }} \\
T_{0}\left(\frac{R_{0}}{r}\right) & R_{\text {in }} \leq r<R_{0} \\
T_{0} e^{\left(R_{0}-r\right) / 4 h} & R_{0} \leq r<R_{\nu} \\
T_{0} e^{\left(R_{0}-r\right) / 4 h}\left(R_{\nu} / r\right) & r \geq R_{\nu}\end{cases} \\
Y(r)= \begin{cases}Y_{\text {in }}+\left(Y_{0}-Y_{\text {in }}\right) \exp \left[-16 \frac{\left(r-R_{\text {in }}\right)^{2}}{R_{\text {in }}^{2}}\right] & r<R_{\text {in }} \\
Y_{0}+\left(Y_{t}-Y_{0}\right) \exp \left[-100 \frac{\left(r-R_{\text {in }}\right)^{2}}{R_{\text {in }}^{2}}\right] & R_{\text {in }} \leq r<R_{t} \\
Y_{t}+\left(Y_{\text {out }}-Y_{t}\right) \frac{r-R_{t}}{R_{\text {out }}-R_{t}} & R_{t} \leq r<R_{\text {out }} \\
Y_{\text {out }} & r \geq R_{\text {out }}\end{cases}
\end{gathered}
$$

We used the following parameters:

$$
\begin{aligned}
R_{\text {in }} & =8 \mathrm{~km} \\
T_{\text {in }} & =15 \mathrm{MeV} \\
Y_{\text {in }} & =0.25 \\
R_{0} & =15 \mathrm{~km} \\
\rho_{0} & =10^{14} \mathrm{~g} \mathrm{~cm}^{-3} \\
T_{0} & =27.5 \mathrm{MeV} \\
Y_{0} & =0.1 \\
R_{\nu} & =21 \mathrm{~km} \\
R_{t} & =25 \mathrm{~km} \\
h & =1 \mathrm{~km} \\
Y_{t} & =0.4 \\
R_{\text {out }} & =30 \mathrm{~km} \\
Y_{\text {out }} & =0.5
\end{aligned}
$$

The parameters were chosen such that the profile coincided well with simulation results produced by DK. The temperature was increased to a maximal realistic value (peak temperature of roughly $50 \mathrm{MeV}$ ) in order to exaggerate any differences with the cooler profile used in the body of this paper. It was found that even this dramatic change in profile increased the bounds by roughly $\Delta(\log \epsilon) \sim 0.1$ and everywhere, the change was 

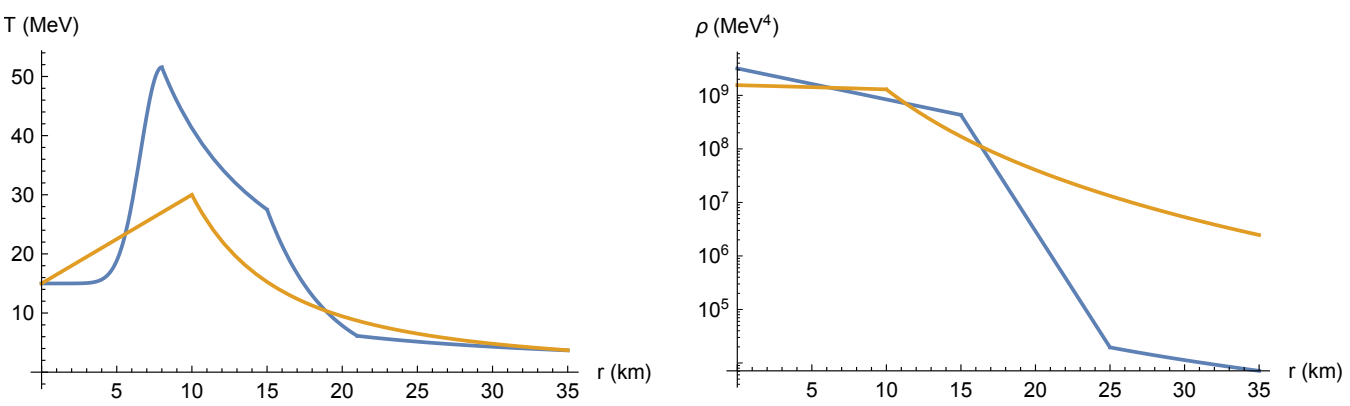

Figure 2. The two SN profiles compared in order to assess the effect of modeling uncertainties on our results. The yellow curve is the profile used to generate our bounds while the blue curve is the one described in this appendix. Though the blue curve reaches a much higher temperature than the yellow curve, the overall effect on our bounds is small. Note that the disagreement in densities at radii outside $20 \mathrm{~km}$ does not cause a significant discrepancy in our results because the majority of production occurs in the dense regions within the core.

$\Delta(\log \epsilon) \leq 0.3$. We conclude that the results presented in this paper are not highly sensitive to the choice of SN profile.

\section{B Dark photon decays with hard photon emission}

Here we describe the calculation of the branching ratio of a dark photon with boost factor $\eta$ to $e^{+} e^{-} \gamma$ with a final state photon energy above some $\omega_{\min }$. At tree level, the averaged matrix element for the decay $A^{\prime} \rightarrow e^{+} e^{-} \gamma$ is

$$
\begin{aligned}
|\overline{\mathcal{M}}|^{2}= & \frac{8 \epsilon^{2} e^{4}}{3\left(m_{23}^{2}-m_{e}^{2}\right)^{2}\left(m^{\prime 2}+m_{e}^{2}-m_{12}^{2}-m_{23}^{2}\right)^{2}} \\
\times & {\left[m^{\prime 6}\left(m_{23}^{2}-3 m_{e}^{2}\right)-m^{\prime 4}\left(3 m_{23}^{4}-6 m_{23}^{2} m_{e}^{2}+m_{12}^{2}\left(m_{23}^{2}-5 m_{e}^{2}\right)+7 m_{e}^{4}\right)\right.} \\
& +m^{\prime 2}\left(m_{12}^{4}\left(m_{23}^{2}-3 m_{e}^{2}\right)+4\left(m_{23}^{2}-m_{e}^{2}\right)^{3}+4 m_{12}^{2}\left(m_{23}^{4}-m_{23}^{2} m_{e}^{2}+2 m_{e}^{4}\right)\right) \\
& -m_{12}^{6}\left(m_{23}^{2}-m_{e}^{2}\right)-2\left(m_{23}^{2}-m_{e}^{2}\right)^{4} \\
& \left.-4 m_{12}^{2} m_{23}^{2}\left(m_{23}^{2}-m_{e}^{2}\right)^{2}-m_{12}^{4}\left(3 m_{23}^{4}-2 m_{23}^{2} m_{e}^{2}+3 m_{e}^{4}\right)\right]
\end{aligned}
$$

where $m_{12}^{2}=(k+q)^{2}$ and $m_{23}^{2}=(k+l)^{2}$ with $k, q$, and $l$ the electron, positron, and photon momenta respectively.

In the dark photon rest frame, the differential partial width of this decay is given by

$$
\frac{d^{2} \Gamma}{d E_{\gamma} d \cos \theta}=\frac{1}{(2 \pi)^{3} 32 m^{\prime 2}} \int d m_{23}^{2}|\overline{\mathcal{M}}|^{2},
$$

with $E_{\gamma}$ the energy of the final-state photon and $\theta$ the angle between the photon momentum and the $\hat{z}$-direction. The integration limits are

$$
\left(m_{23}^{2}\right)_{\max / \min }=\left(E_{2}^{*}+E_{3}^{*}\right)^{2}-\left(\sqrt{E_{2}^{* 2}-m_{e}^{2}} \mp E_{3}^{*}\right)^{2}
$$


with

$$
E_{2}^{*}=\frac{m_{12}}{2} \quad \text { and } \quad E_{3}^{*}=\frac{m^{\prime 2}-m_{12}^{2}}{2 m_{12}} .
$$

If we require that the energy of the photon is above $\omega_{\min }$ in the frame in which the dark photon has a boost $\eta$ in the $\hat{z}$-direction, this translates to a minimum energy

$$
\left(E_{\gamma}\right)_{\min }=\frac{\omega_{\min }}{\eta+\cos \theta \sqrt{\eta^{2}-1}}
$$

in the rest frame. Using this constraint, we can integrate eq. (B.2) over $E_{\gamma}$ and $\theta$ to obtain the partial width of the boosted dark photon with a final state photon with energy above $\omega_{\min }$.

Open Access. This article is distributed under the terms of the Creative Commons Attribution License (CC-BY 4.0), which permits any use, distribution and reproduction in any medium, provided the original author(s) and source are credited.

\section{References}

[1] R. Essig et al., Working group report: new light weakly coupled particles, in Proceedings, 2013 Community Summer Study on the Future of U.S. Particle Physics: Snowmass on the Mississippi (CSS2013), Minneapolis, MN, U.S.A., 29 July-6 August 2013 [arXiv:1311.0029] [INSPIRE].

[2] J. Alexander et al., Dark sectors 2016 workshop: community report, arXiv: 1608.08632 [INSPIRE].

[3] G.G. Raffelt, Stars as laboratories for fundamental physics, Chicago Univ. Pr., Chicago, IL, U.S.A. (1996) [INSPIRE].

[4] B. Holdom, Two U(1)'s and $\epsilon$ charge shifts, Phys. Lett. B 166 (1986) 196 [INSPIRE].

[5] J.H. Chang, R. Essig and S.D. McDermott, Revisiting supernova 1987A constraints on dark photons, JHEP 01 (2017) 107 [arXiv:1611.03864] [InSPIRE].

[6] E. Hardy and R. Lasenby, Stellar cooling bounds on new light particles: plasma mixing effects, JHEP 02 (2017) 033 [arXiv: 1611.05852] [INSPIRE].

[7] E. Rrapaj and S. Reddy, Nucleon-nucleon bremsstrahlung of dark gauge bosons and revised supernova constraints, Phys. Rev. C 94 (2016) 045805 [arXiv: 1511.09136] [INSPIRE].

[8] H. An, M. Pospelov and J. Pradler, New stellar constraints on dark photons, Phys. Lett. B 725 (2013) 190 [arXiv:1302.3884] [INSPIRE].

[9] E. Braaten and D. Segel, Neutrino energy loss from the plasma process at all temperatures and densities, Phys. Rev. D 48 (1993) 1478 [hep-ph/9302213] [INSPIRE].

[10] T. Siegert et al., Gamma-ray spectroscopy of positron annihilation in the Milky Way, Astron. Astrophys. 586 (2016) A84 [arXiv:1512.00325] [INSPIRE].

[11] N. Prantzos et al., The $511 \mathrm{keV}$ emission from positron annihilation in the galaxy, Rev. Mod. Phys. 83 (2011) 1001 [arXiv: 1009.4620] [INSPIRE].

[12] J.F. Beacom and H. Yuksel, Stringent constraint on galactic positron production, Phys. Rev. Lett. 97 (2006) 071102 [astro-ph/0512411] [INSPIRE]. 
[13] P. Sizun, M. Casse and S. Schanne, Continuum gamma-ray emission from light dark matter positrons and electrons, Phys. Rev. D 74 (2006) 063514 [astro-ph/0607374] [INSPIRE].

[14] J.H. Groh, G. Meynet, C. Georgy and S. Ekstrom, Fundamental properties of core-collapse supernova and GRB progenitors: predicting the look of massive stars before death, Astron. Astrophys. 558 (2013) A131 [arXiv:1308.4681] [INSPIRE].

[15] M. Matsuura et al., Herschel SPIRE and PACS observations of the red supergiant VY CMa: analysis of the molecular line spectra, Mon. Not. Roy. Astron. Soc. 437 (2014) 532 [arXiv: 1310.2947].

[16] S.M. Adams, C.S. Kochanek, J.F. Beacom, M.R. Vagins and K.Z. Stanek, Observing the next galactic supernova, Astrophys. J. 778 (2013) 164 [arXiv:1306.0559] [INSPIRE].

[17] S.-C. Yoon, S.E. Woosley and N. Langer, Type Ib/c supernovae in binary systems I. Evolution and properties of the progenitor stars, Astrophys. J. 725 (2010) 940 [arXiv: 1004.0843] [INSPIRE].

[18] P.A. Crowther, Physical properties of Wolf-Rayet stars, Ann. Rev. Astron. Astrophys. 45 (2007) 177 [astro-ph/0610356] [INSPIRE].

[19] O. Graur et al., LOSS revisited - II: the relative rates of different types of supernovae vary between low- and high-mass galaxies, Astrophys. J. 837 (2017) 121 [arXiv:1609.02923] [INSPIRE].

[20] K.-W. Chan and R.E. Lingenfelter, Positrons from supernovae, Astrophys. J. 405 (1993) 614.

[21] P. Fraser, Positrons and positronium in gases, in Advances in atomic and molecular physics, Elsevier, The Netherlands (1968), pg. 63.

[22] H.-T. Janka, B. Müller, F.S. Kitaura and R. Buras, Dynamics of shock propagation and nucleosynthesis conditions in O-Ne-Mg core supernovae, Astron. Astrophys. 485 (2008) 199 [arXiv:0712.4237] [INSPIRE].

[23] D. Kazanas, R.N. Mohapatra, S. Nussinov, V.L. Teplitz and Y. Zhang, Supernova bounds on the dark photon using its electromagnetic decay, Nucl. Phys. B 890 (2014) 17 [arXiv: 1410.0221] [INSPIRE].

[24] T. Piran, Gamma-ray bursts and the fireball model, Phys. Rept. 314 (1999) 575 [astro-ph/9810256] [INSPIRE].

[25] P. Meszaros, Gamma-ray bursts, Rept. Prog. Phys. 69 (2006) 2259 [astro-ph/0605208] [INSPIRE].

[26] E.W. Kolb and M.S. Turner, Limits to the radiative decays of neutrinos and axions from gamma-ray observations of SN 1987a, Phys. Rev. Lett. 62 (1989) 509 [InSPIRE].

[27] P. Podsiadlowski, The progenitor of SN 1987a, Publ. Astron. Soc. Pacific 104 (1992) 717.

[28] J.F. Beacom, The diffuse supernova neutrino background, Ann. Rev. Nucl. Part. Sci. 60 (2010) 439 [arXiv: 1004.3311] [INSPIRE].

[29] K. Watanabe, The MeV cosmic gamma-ray background measured with SMM, AIP Conf. Proc. 510 (2000) 471.

[30] R.L. Kinzer, G.V. Jung, D.E. Gruber, J.L. Matteson and L.E. Peterson, Diffuse cosmic gamma radiation measured by HEAO 1, Astrophys. J. 475 (1997) 361.

[31] A.W. Strong et al., Diffuse galactic hard X-ray and low-energy gamma-ray continuum, Astron. Astrophys. Supp. 120 (1996) 381. 
[32] A. Fradette, M. Pospelov, J. Pradler and A. Ritz, Cosmological constraints on very dark photons, Phys. Rev. D 90 (2014) 035022 [arXiv: 1407.0993] [InSPIRE].

[33] J. Redondo and M. Postma, Massive hidden photons as lukewarm dark matter, JCAP 02 (2009) 005 [arXiv:0811.0326] [INSPIRE].

[34] R. Essig, E. Kuflik, S.D. McDermott, T. Volansky and K.M. Zurek, Constraining light dark matter with diffuse X-ray and gamma-ray observations, JHEP 11 (2013) 193 [arXiv: 1309.4091] [INSPIRE]. 